\title{
Optimization of Denoising Technique of Phonocardiography Signal By Using Discrete Wavelet Transform
}

\author{
Dyah Kurniawati Agustika $^{1}$, Sumarna $^{1}$, Agus Purwanto $^{1}$, Juli Astono ${ }^{1}$ \\ \{dyah_kurniawati@uny.ac.id, sumarna@uny.ac.id, agus_purwanto@uny.ac.id,juli_astono@uny.ac.id\} \\ Department of Physics Education, Universitas Negeri Yogyakarta, Yogyakarta, Indonesia ${ }^{1}$
}

\begin{abstract}
The purpose of this research is to design and build Phonocardiography (PCG) system that can record the human heartbeat representatively and reliably, which is a device that can record a lot of information contained in a heartbeat. This research begins by designing and realizing devices that can record acoustic heartbeat signals. The PCG signal is easy to affect by noise such as stethoscope movement, the friction of stethoscope and chest, etc.. Therefore, signal analysis process such as denoising is needed. Denoising can be performed by using a discrete wavelet transform (DWT). In this research, the kind of wavelet that were used are coiflet1, symlet1, symlet2, daubechies3, and daubechies 4 . This wavelet type is compared with the original signal, and the mean square error (MSE) calculated. From the analysis results, the type of wavelet that gives the smallest MSE is symlet 2 indicating that wavelet most resembles the original signal compared with the other two wavelets. The result of character extraction will be used in subsequent research to be input in a pattern recognition system.
\end{abstract}

Keywords: Phonocardiography, Signal Denoising, Discrete Wavelet Transform.

\section{Introduction}

Heart disease can be detected from the sound produced by the heart because the sound describes the physiological condition and carries information on the pathological state of the heart. The frequency of heart signals ranges from 10 to $250 \mathrm{~Hz}$, and if there are abnormalities in the heart, a higher frequency can be heard[1][2]. The technique of analyzing heart rate frequency spectrum based on graphs of recorded heart mechanical pulses using a stethoscope is known as phonocardiography[3][4]. Phonocardiography is a device that is able to record heart sounds and display signals obtained in the form of graphs drawn with signal amplitude against time[1]. The electronic stethoscope used by PCG converts the heart's voice signal into an electrical form using a microphone. The advantage of PCG from the detection of other heart disorders is its easy use (unlike an electrocardiograph that requires special expertise) and its low cost. Heart signal frequencies that are quite low, between 10 and $250 \mathrm{~Hz}$ are very weak to be detected and can be easily disturbed by noise[1][2] [5]. Therefore, PCG signals are very susceptible to various sounds produced by patient movements, stethoscope movements used to obtain PCG signals, pulmonary sounds, and external sounds of the surrounding environment. In addition, pathology can cause extra noise in the PCG signal, which is called a murmur. Noise can make PCG analysis difficult. Thus, it is important to analyze heart sound signals accurately and eliminate signals that interfere successfully during pre-processing[2][3][5]. 
Several algorithms for denoising PCG signals in the frequency domain have been carried out. Conventional filters are limited to suppress noise coming out of the signal component frequency bands[5]. FFT (Fast Fourier Transform) analysis can describe the frequency of the heart sound. However, the FFT technique has the disadvantage of transforming the signal into the frequency domain; the time information is lost. In addition, FFT analysis only matches stationary signals, while the heart rate shows changes marked by time and frequency, and is not a stationary signal. Heart rate shows sudden changes in frequency and transients and power spectrum analysis of limited values. The solution is to use wavelet transforms. Wavelet Transform is a tool that can be used to denoise PCG signals because wavelet allows doing multiresolution analysis, which separates the signal into high frequency and low-frequency component and helps to achieve time and frequency localization. The wavelet algorithm processes data on different scales or resolutions[3]. In this research we choose various wavelets on PCG signals and attempts were made to find wavelets that resambles the original signal the most hence it can be used to denoise the signal.

The method that can be used in the denoising process is a discrete wavelet transformation because wavelets allow processing of data on different scales or resolutions[3]. PCG output signal can be described into the base function. For DWT, the basic function used to decipher the signal is the scaling function and the wavelet function. The form of these two basic functions is written in the equation (1) and (2)

$$
\begin{aligned}
& \varphi_{j, k}(t)=2^{j / 2} \varphi\left(2^{j} t-k\right) \\
& \psi_{j, k(t)}=2^{j / 2} \psi\left(2^{j} t-k\right)
\end{aligned}
$$

Factor $2^{j / 2}$ is the base normalization factor, $k$ is a shift, or translation factor and $j$ is a scale factor. The original signal can be represented by a wavelet base function with different translations and scales. The $x(t)$ signal described as a linear combination of scaling functions and wavelet functions is written in the equation (3)

$$
x(t)=\sum_{k} a_{j_{0}}(k) 2^{j_{0} / 2} \varphi\left(2^{j_{0}} t-k\right)+\sum_{k} \sum_{j=j_{0}}^{\infty} d_{j}(k) 2^{j / 2} \psi\left(2^{j} t-k\right)
$$

$a_{j_{0}}$ is called the approximation coefficient, $d_{j}$ is called the detail coefficient.

The process of decoding signals into approximation and detail components using a base scaling function and wavelet function is known as wavelet decomposition[6][7]. The approximation is the result of filtering a low-frequency filter that represents the identity of the original signal, while the detailed is the result of filtering the high-frequency filter. The approximation component can be passed to the next filter to obtain approximation and detail components at a higher level. In the process, there will be a decrease in the sample because some components (samples) of the signal are removed. In this operation, convolution is only done on even data or can be taken only even. This operation is called downsampling. The number of samples in this process output is part of the number of input samples. This process is represented by $2 \downarrow$ as shown in Figure 1 . 


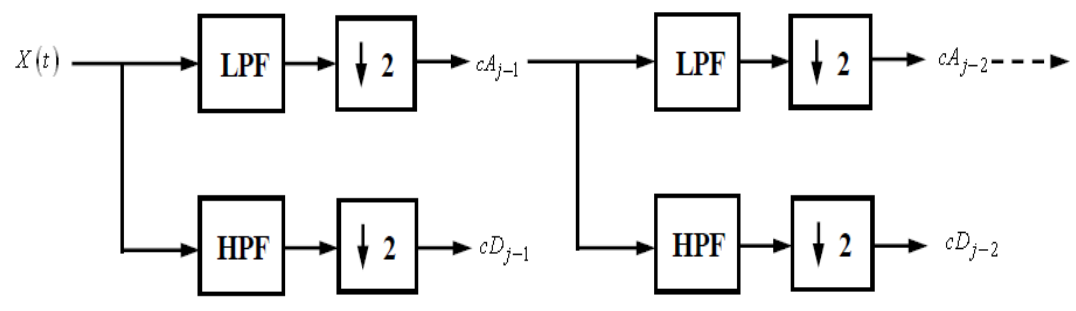

Fig. 1. Multilevel Wavelet Decomposition [8]

The approximation component represents the original signal. By ignoring the detailed components and passing the approximation component into the next branched filter as shown in Figure 3.2, the original signal can be decomposed into the multilevel approximation [9].

\section{Research Method}

The steps in this research depicts in Figure 1.

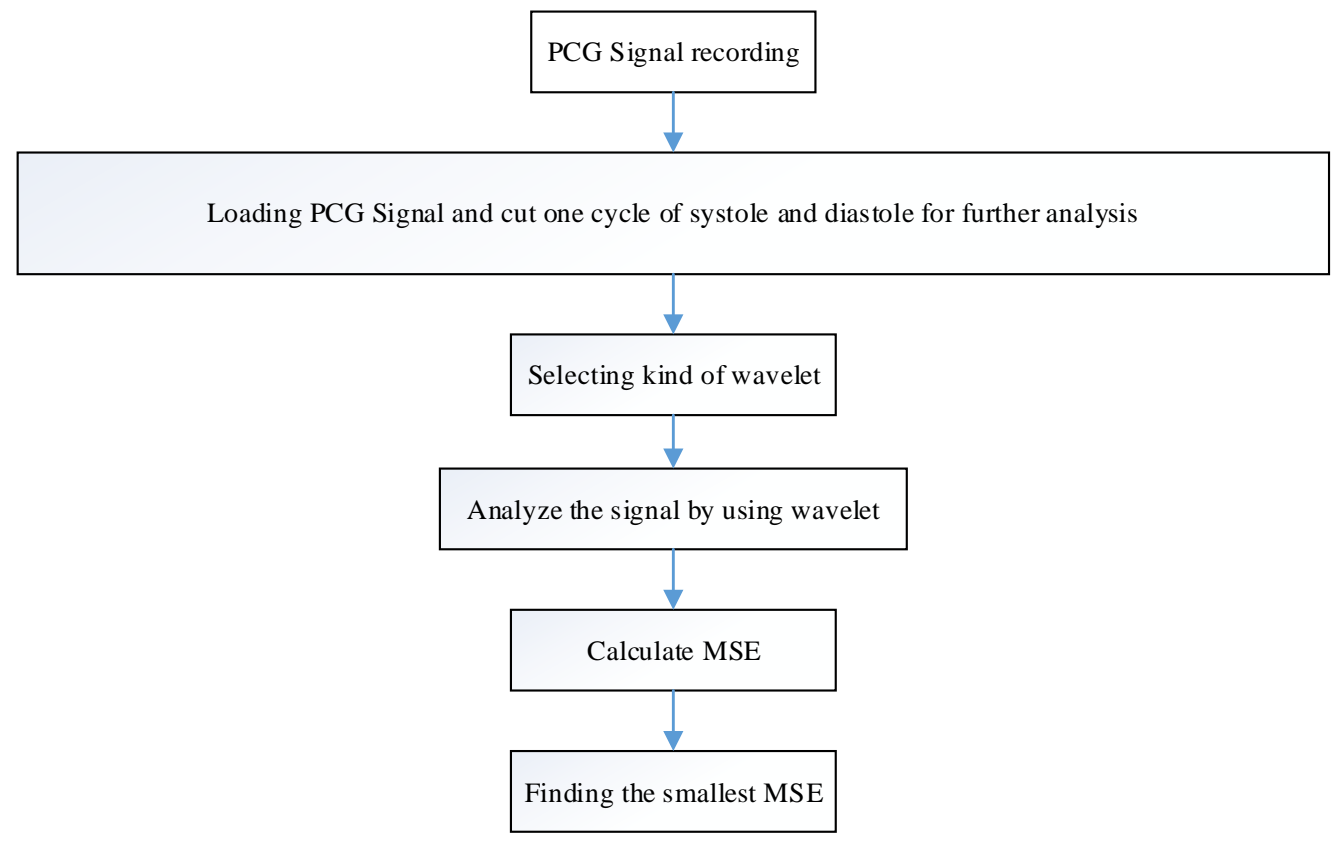

Fig. 2.. Flowchart of research method

Heart beat recording was done by using PCG and the recording results depicted in Figure. 3

The next step is to cut the signal to one cycle of systole and diastole. Figure 4 illustrates one cycle of systole and diastole 


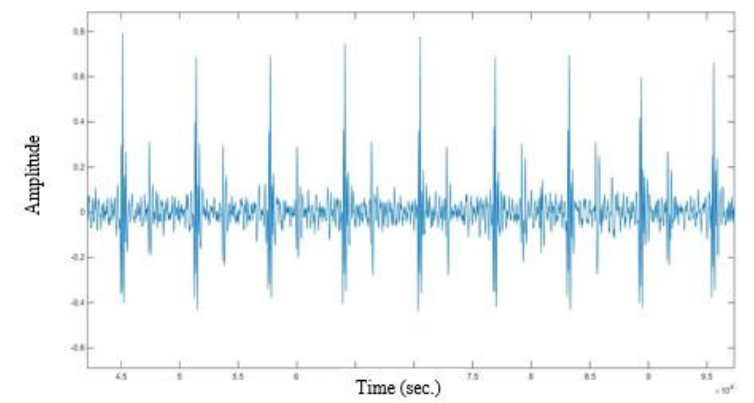

Fig. 3. Heartbeat signal recording [4]

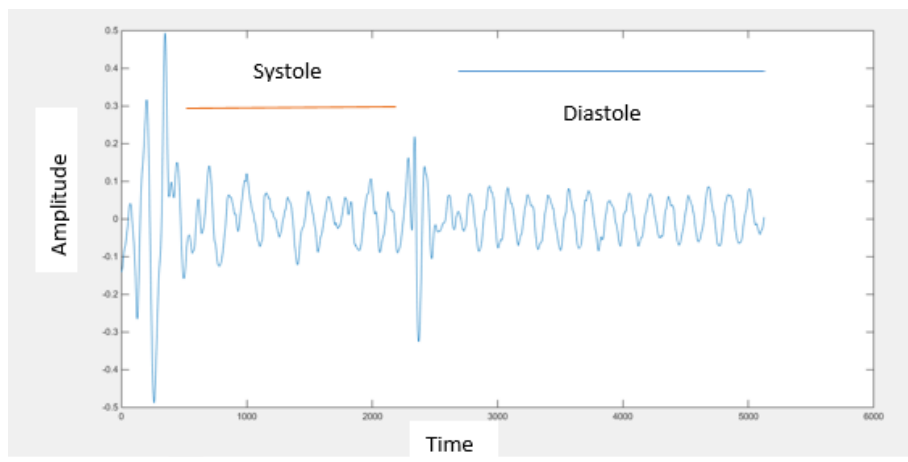

Fig. 4. One cycle of systole and diastole

The next step is to choose the kind of wavelet that represent the signal. From many wavelets, we choose three different wavelets and then compare it to the original signal then we count the mean square error. Three kinds of wavelet depicted in Figure 5.
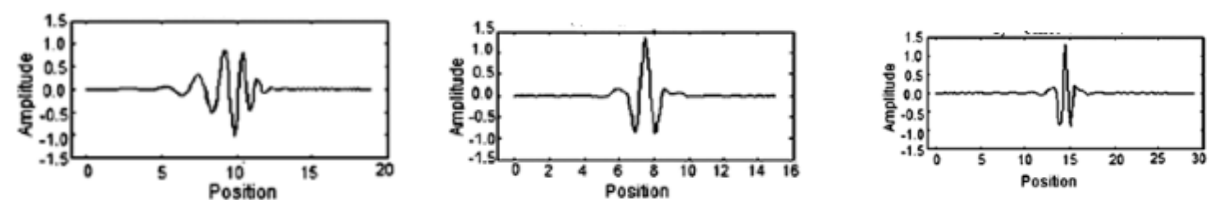

Fig. 3. Three kinds of wavelet (a) Daubechies (b) Symlet (c) Coiflet [9].

The recorded data of heartbeat signal then cut in the part of systole and diastole. 


\section{Discussion}

After choosing the data then the next step is to choose the kind of wavelet. Here we use coiflet1, symlet2, symlet3, daubechies3, daubechies4. This kind of wavelet is orthogonal. Optimization is done by comparing the mean square error (MSE) value of the wavelet reconstruction result to PCG signal. The most suitable type of wavelet is one that has the smallest MSE value.

The PCG output signal is processed by using DWT to level one then each decomposition coefficient, cA1, cD1 are summed again to reconstruct the PCG output signal. After the reconstruction process, MSE can be found from equation (4)[10].

$$
M S E=\frac{(S-A)^{2}}{n}
$$

Type of wavelet that has the smallest value of MSE is chosen to be the wavelet to denoise the PCG signal because it is the wavelet that most similar to the original signal

Table 1. MSE value of each wavelet.

\begin{tabular}{cc}
\hline Kind of wavelet & MSE \\
\hline Coif1 & $6.2316 \times 10-31$ \\
Sym2 & $4.0919 \times 10-31$ \\
Sym3 & $3.3182 \times 10-28$ \\
Db3 & $3.3182 \times 10-28$ \\
Db4 & $1.2938 \times 10-29$ \\
\hline
\end{tabular}

From the Table 1, it can be seen that the wavelet that resembles the signal the most is Symlet2 because the MSE is the lowest compare with other wavelets.

\section{Conclusion}

From the data analysis, it can be concluded that the best wavelet to represent the PCG signal in this research is symlet1 with the lowest score of MSE. After choosing the kind of wavelet the next research is to denoising the PCG signal, The best wavelet is sym2.

\section{References}

[1] L. Bahekar and A. Misal, "Heart Sounds Segmentation Analysis Using Daubechies Wavelet ( db )," vol. 2, no. 2, pp. 1-6, 2014.

[2] A. Misal and G. R. Sinha, "DENOISING OF PCG SIGNAL BY USING WAVELET TRANSFORMS," vol. 4, no. 1, pp. 46-49, 2012.

[3] G. Mishra, K. Biswal, and A. K. Mishra, "DENOISING OF HEART SOUND SIGNAL 
USING WAVELET TRANSFORM,” pp. 719-723, 2013.

[4] J. Astono, A. Purwanto, and D. K. Agustika, "The Improvement of Phonocardiograph Signal ( PCG ) Representation Through the Electronic Stethoscope," no. September, pp. 19-21, 2017.

[5] P. Kumar and A. Kumar, "Biomedical Signal Processing and Control An adaptive thresholding method for the wavelet based denoising of phonocardiogram signal," Biomed. Signal Process. Control, vol. 38, pp. 388-399, 2017.

[6] "R.M. Rao, A.S. Bopadipkar, "Wavelet Transform: Introduction to Theory and Applications" Massachusets,Addison-Wesley, 1998.

[7] D. K. Agustika and K. Triyana, "Application of Principal Component Analysis and Discrete Wavelet Transform in Electronic Nose for Herbal Drinks Classification," vol. 170012, pp. 1-7, 2016.

[8] E. PHAISANGITTISAGUL, "Signal Processing using Wavelets for Enhancing Electronic Nose Performance," North Carolina State University, 2007.

[9] "Multi-scale analysis of well-logging data in petrophysical and stratigraphic correlation," no. August, 2016.

[10] M. V. Shcherbakov and A. Brebels, "A Survey of Forecast Error Measures,” vol. 24, no. 4, pp. 171-176, 2013. 